2017-03-07

\title{
On the harmonization of methods for measuring the occurrence, fate and effects of microplastics
}

\section{Rochman, CM}

http://hdl.handle.net/10026.1/9207

\subsection{9/c7ay90014g}

Analytical Methods

Royal Society of Chemistry (RSC)

All content in PEARL is protected by copyright law. Author manuscripts are made available in accordance with publisher policies. Please cite only the published version using the details provided on the item record or document. In the absence of an open licence (e.g. Creative Commons), permissions for further reuse of content should be sought from the publisher or author. 
This is a pre---publication version. Readers are advised to refer to the final published version.

\section{On the harmonization of methods for measuring the occurrence, fate and effects of microplastics}

Chelsea M. Rochman, ${ }^{\mathrm{a}}$ Fiona Regan $^{\mathrm{b}}$ and $\underline{\text { Richard C. Thompson }}^{\mathrm{c}}$

http://pubs.rsc.org/en/content/articlelanding/2017/ay/c7ay90014g\#!divAbstract

On the harmonization of methods for measuring the occurrence, fate and effects of microplastics

Chelsea M. Rochman ${ }^{\mathrm{a}}$, Fiona Regan ${ }^{\mathrm{b}}$, Richard C. Thompson ${ }^{\mathrm{c}}$

Author Affiliations:

${ }^{a}$ University of Toronto, Department of Ecology and Evolutionary Biology, St. George, Ontario, Canada

${ }^{\mathrm{b}}$ Dublin City University, Department of Chemical Sciences, Dublin, Ireland.

${ }^{c}$ University of Plymouth, Faculty of Science and Engineering, Plymouth, PL4 8AA, United Kingdom.

One-sentence description: This editorial is the introduction to this themed issue, which we hope facilitates discussions that lead toward harmonized methods that are informed by hypotheses, and ultimately produce data that can be synthesized and used to inform effective local and global policies that prevent and mitigate microplastics.

\section{Main text:}

We are delighted to introduce this themed issue on microplastics in the environment in Analytical Methods. The motivation for such an issue was based on researchers and statutory agencies around the world calling for the standardization or harmonization of methods to facilitate more effective sampling and analyses of microplastics. The editors of this volume have participated in numerous meetings where there has been in depth discussion about the "right" standard method. As a consequence, the objective of this volume was to compile a single text for a government agency, commercial laboratory or research group to find reviews on the various methods for different environmental sample types and hypotheses as well as introductions for new methods and ideas. Our overarching goal is for this compilation of work to help guide and/or facilitate the harmonization, rather than standardization, of methods so that data can be useful at different scales - e.g., locally, globally and across disciplines. 
Microplastics, defined by many government agencies as small pieces of plastic litter less than 5 $\mathrm{mm}$ in size, have been reported in marine and freshwater habitats worldwide in a range of diverse media (e.g., sediment, water, biota, ice and air). Microplastics are complex, and just like chemical contaminants they are made up of many different constituent chemical mixtures and enter the environment from a number of different sources. Based on similarities with chemical contaminants, we can learn from methods that identify and quantify chemicals in the environment. But, microplastics are a highly heterogeneous mixture of different types of solid polymers with different densities, sizes and shapes. As such, we must make decisions regarding sampling and extraction methodologies that accurately capture and identify this unique material.

Partly because microplastics were only recently described and because it remains an evolving field, scientists do not have a go-to or standardized methodology for the extraction, quantification, characterization and toxicity of the debris. As a consequence, the data related to microplastics around the world is based on a variety of sampling and extraction equipment and methodology that capture and identify microplastics of differing size ranges and report data in a range of units (e.g., pieces or mass per $\mathrm{km}^{2}$ and/or $\mathrm{m}^{3}$ ). As monitoring programs for microplastics emerge, spearheaded by governments and agencies around the world, and as scientists and managers attempt to synthesize data from specific regions or globally to help reveal largescale spatial and temporal trends, researchers are increasingly calling for consistency in methods for sampling, extracting and reporting microplastics.

In this themed issue, readers will find a compilation of editorials, reviews and original research papers covering an assortment of topics relevant to the methods that are used to answer questions about sources, contamination and effects of microplastics. These include discussions about methods for 1) sampling, extracting and identifying microplastics in different environmental media (e.g., water, sediment, animals), 2) citizen science, 3) designing hypothesis- or objectivedriven sampling regimes to fit the research question, monitoring program and/or management, 4) quantifying the occurrence and fate of chemicals and fouling communities on microplastic debris, 5) assessing the risks associated with microplastics and 6) social and behavioral research.

There are numerous different approaches for sampling, extracting and identifying the diverse mixture of microplastics, and we argue that there is no "right" method and that method standardization is not currently feasible or advisable. When choosing a method, there will always be a tension between accuracy, precision and feasibility. Some methods may be designed to be more technical and others may be created to facilitate citizen science. In general, sampling designs should be created in a way that answer the questions and relate to the hypotheses of the specific research. Such questions may inform sampling locations, equipment and number of replicates. In all cases, we need to consider and optimize in relation to several overarching issues. First, it is important to consider temporal and spatial scales understanding that microplastic distribution is highly variable across space and time. Second, it is important to choose a method that will capture the size-range of interest, effectively extract microplastics from the media without dissolving or melting the material of interest and identify and confirm the many different types of microplastics. The chosen method will vary based on the media of interest. We often discuss sampling in water versus sediments versus animals. It is important to note that there are other matrices that are also important. For example, to understand sources, it is useful to harmonize methods to extract, identify and quantify microplastics in wastewater, 
sewage sludge and air and to understand the fate, it is useful to harmonize methods in polar ice and marine snow. An overriding priority is to describe the data collected in such a way as to prevent ambiguity. What may be most important in this case are units, it is important to use consistent units so that we can synthesize data across studies to ask questions about broader contamination.

While the above discussion on quantification of microplastics may seem the most common, it is also important to design methods that help us understand other questions around the issue. In particular, questions related to the potentially harmful impacts of microplastics. It is well-known that plastics are associated with unique cocktails of chemicals and that they increase their load of chemicals when they enter aquatic habitats via sorption. In addition, they gain a fouling community that hitchhikes on its surface. Methods related to the fate and occurrence of these chemicals and communities are useful to understand issues related to fate and effects. As plastics transport around the ocean, they may be a vector of unique chemicals and/or biological communities. When it comes to the effects of microplastics, effects may vary by type based on their unique chemistry, fouling community or simply shape and size. In general, questions related to effects are becoming increasingly important due to widespread contamination of microplastics. As such, the need for accepted methods for measuring effects and using them in assessments of risk is increasingly brought to the table.

Last but not least is the human dimension. At the end of the day, we are the source of microplastics to the environment and the ones that are capable of preventing and cleaning up our litter. To not include social and behavioral research in our themed issue would be a disservice to management agencies. To understand how microplastics become litter and to reduce the pollution, we need to also apply social and behavioral research methods. In this issue, the human dimension is described as three-pronged: humans contribute to the microplastic problem, humans may experience negative impacts from the microplastic problem and humans ultimately have to be the ones to solve the microplastic problem.

The topic of microplastics is no longer new and agencies around the world are adding microplastics to their lists of contaminants of concern. Right now, government scientists are designing and implementing monitoring programs and agencies are soliciting proposals for research that will help them better understand microplastic contamination. At this stage, it is crucial that we initiate a dialogue that is iterative. Historically the rationale for monitoring has been because there is a concern. Whether concerns are around sources and fate of microplastics, negative impacts to wildlife or food safety and security, we must recognize that our understanding is still incomplete. Thus, it is imperative that we design protocols around clear objectives and hypotheses. We hope that this compilation of work helps inform discussions that lead to effective, harmonized and widely-accepted methods for gathering and reporting data. Such a harmonization of methods will allow us to synthesize data in individual locations and around the world to help inform local and global policies that prevent and mitigate the escalating contamination from plastic debris worldwide. 